\title{
Safety and effectiveness of hyaluronic acid dermal filler in correction of moderate-to-severe nasolabial folds in Chinese subjects
}

This article was published in the following Dove Medical Press journal: Clinical, Cosmetic and Investigational Dermatology

\author{
Xia Dai' \\ $\mathrm{Li} \mathrm{Li}^{2}$ \\ Ward Peterson ${ }^{3,4}$ \\ Renate R Baumgartner ${ }^{3}$ \\ Jinmei Huang ${ }^{5}$ \\ Alexandra Baer-Zwick ${ }^{6}$ \\ Sonja Hoeller ${ }^{3}$ \\ Zrinka Ivezic-Schoenfeld ${ }^{3}$ \\ Martin Prinz ${ }^{3}$ \\ 'Department of Plastic and \\ Reconstructive Surgery, The First \\ Hospital Affiliated to Army Medical \\ University, Chongqing, China; \\ ${ }^{2}$ Department of Dermatology, \\ West China Hospital, Sichuan \\ University, Sichuan, China; ${ }^{3}$ Clinical \\ Development, CROMA-PHARMA \\ GmbH, Leobendorf, Austria; ${ }^{4}$ Ward \\ Peterson Consulting, Limited Liability \\ Company, Morrisville, NC 27560, USA \\ ${ }^{5}$ Qualtech Consulting Corporation, \\ Taipei, Taiwan; ${ }^{6}$ Regulatory Affairs, \\ CROMA-PHARMA GmbH, \\ Leobendorf, Austria
}

Correspondence: Martin Prinz Clinical Development, CROMAPHARMA $\mathrm{GmbH}$, Industriezeile 6 , A-2 100 Leobendorf, Austria Tel +00432262 68468109 Email martin.prinz@croma.at
Background: Modified sodium hyaluronate gel for injection, Princess ${ }^{\circledR}$ VOLUME (PV), has been on the European market since 2009 to correct deeper wrinkles and folds, increasing or restoring volume of the face, and remodeling facial contours.

Objective: To evaluate the safety and effectiveness of PV in correction of moderate-to-severe nasolabial folds (NLF) in Chinese subjects.

Methods: In this prospective, split-face, randomized, evaluator and subject-blinded, multicenter, noninferiority trial, 120 subjects were randomized to bilateral NLF treatment with PV administered in one NLF and Restylane ${ }^{\circledR}$ (RL) administered in the other NLF. NLFs were evaluated using the validated 5-point Wrinkle Severity Rating Scale with scores ranging from $1=$ none (no visible NLF) to $5=$ very severe (extremely deep and long NLF). Response was defined as $\geq 1$ point improvement at Week 24 assessed by the blinded independent review committee (IRC) and the reduction of NLF severity, assessed by subjects and IRC based on the Global Aesthetic Improvement Scale.

Results: Among the 115 subjects who completed the study, median initial and touch-up volumes $(\mathrm{mL})$ were 1.00 for both groups with a maximum dosage per NLF of 2.00 and a minimum of 0.30 for PV and 0.60 for RL. At week 24, the Wrinkle Severity Rating Scale improvement rate, as assessed by the IRC, reached $68.70 \%$ for PV and $52.17 \%$ for RL. The results indicate that $\mathrm{PV}$ is noninferior to RL $(p<0.001)$. Most frequently reported adverse events for both devices were injection site swelling and procedural pain. The severity of the majority of the adverse events was mild.

Conclusion: This study confirms that PV is a safe and effective treatment for the correction of moderate-to-severe NLFs in Chinese subjects.

Keywords: nasolabial folds, Princess ${ }^{\circledR}$, Princess ${ }^{\circledR}$ VOLUME, effectiveness, safety, treatment satisfaction

\section{Introduction}

Facial wrinkles and folds are the natural results of the aging process. ${ }^{1}$ The decline of endogenous hyaluronic acid (HA) results in a less hydrated and consequently less elastic skin. ${ }^{2}$ In the past 20 years, it has become increasingly common to use injectable soft tissue fillers to correct facial defects. ${ }^{2,3}$ HA-based dermal fillers have been proven to be safe, effective, and easy to use, thus becoming the most popular type of dermal fillers and widely used. ${ }^{4,5}$

According to the statistics by the official website of the American Society for Aesthetic Plastic Surgery (ASAPS), the number of cases receiving HA injection for 
filling treatment was up to $>2.49$ million in 2016, ranking second in the USA, only next to Botulinum toxin Type A in nonsurgical cosmetic procedures. ${ }^{6}$

Princess ${ }^{\circledR}$ VOLUME (PV), manufactured by CROMA $\mathrm{GmbH}$, is a highly cross-linked, viscoelastic, HA-injectable gel implant used for esthetic treatment, which received the CE mark in 2009. It has obtained market approval in the European Union and in 30 additional countries worldwide. PV filler is based on biofermentative HA crosslinking with 1,4-butanediol diglycidyl ether, which leads to an increased tissue residence.

To obtain China Food and Drug Administration approval, this clinical investigation evaluated the safety and effectiveness of PV for correction of moderate-to-severe nasolabial folds (NLFs) in Chinese subjects.

\section{Methods}

\section{Study design}

This was a prospective, split-face, randomized, evaluator and subject-blinded, multicenter, noninferiority trial. Eligible subjects were randomized to bilateral NLF treatment with the investigational medical device PV administered in one NLF and the comparator medical device Restylane ${ }^{\circledR}$ (RL; Q-Med AB, Uppsala, Sweden) administered in the other NLF. The volume injected was chosen by the investigator and depended on the depth of wrinkle to be corrected. One touch-up treatment was possible at 2 weeks after initial treatment, if, per investigator's opinion, optimal correction was not achieved.

The study protocol conformed to the ethical guidelines of the Declaration of Helsinki and was approved by independent ethics committees of West China Hospital Sichuan University Clinical Trials and Biomedical Ethics Committee and the Ethics committee of the First Affiliated Hospital of Third Military Medical University.

\section{Subjects}

The subjects were required to be aged between 30 and 65 years, without gender preference, and with approximately symmetrical NLFs of moderate (3) or severe (4) wrinkle severity score, as evaluated on the 5-point Wrinkle Severity Rating Scale (WSRS). Subjects signed informed consent before any procedure could take place and were excluded if they were pregnant, had serious skin diseases, facial surgery, or implantation of dermal fillers in the nasolabial region within the last 24 months, any type of facial or cosmetic procedure within the last 3-12 months, diabetes mellitus, uncontrolled systemic diseases, or tendency to keloid formation or were not suitable according to judgment of the investigator.

\section{Assessments}

Assessment for effectiveness was performed by the Independent Review Committee (IRC) and the investigators using the WSRS, and it was performed at 4, 12, and 24 weeks after injection. ${ }^{7}$ Subjects and the IRC independently evaluated NLF improvement using the Global Aesthetic Improvement Scale (GAIS) at 4, 12, and 24 weeks after injection. ${ }^{8}$ The GAIS is a 5-point scale used for rating the esthetic improvement of NLFs compared to pretreatment appearance. The ratings are worse ( 5 points), no change ( 4 points), improved ( 3 points), much improved ( 2 points), and very much improved (1 point).

Safety was evaluated throughout the whole study duration from the first application to the last visit at week 52. Subjects were also encouraged to spontaneously report adverse events (AEs) and were asked for any AE at each study visit.

\section{Measures and endpoints}

The primary efficacy endpoint was the WSRS improvement rate for each NLF after 24 weeks relative to baseline, defined as the percentage of subjects who showed $\geq 1$-point improvement for each NLF. The primary endpoint was a noninferiority comparison of responder rates for PV vs RL at 24 weeks. The secondary efficacy endpoints included the improvement rate based on subjects' and IRC's assessments of NLF improvement by using GAIS 4, 12, and 24 weeks after injection of PV compared with RL.

\section{Statistical analysis}

The clinical investigation employs a noninferiority test for the primary endpoint analysis with a noninferiority margin of the WSRS improvement rate of $15 \%$ (Wald-type asymptotic test). ${ }^{9}$

The primary efficacy endpoint is the WSRS improvement rate at week 24 (Visit 7) relative to baseline (Visit 2).

"Effectiveness" is defined as the WSRS improvement rate $\geq 1$. Subjects' evaluation of NLFs by using GAIS at 24 weeks compared to baseline was summarized, and the $95 \%$ CI was calculated for the GAIS scores for PV and RL.

The primary efficacy analysis was performed in the per-protocol (PP) population, defined as all subjects who were randomized, received treatment, had primary efficacy assessments at 24 weeks, and did not have any major protocol deviations.

Safety analyses were performed in the safety analysis (SA) population, defined as all subjects who received at least one treatment.

The full analysis set (FAS) comprises randomized subjects who received at least one administration of the investigational 
medical device and the control medical device and who had at least one evaluation of the efficacy endpoint at either 4 , 12 , or 24 weeks (Visits 5, 6, and 7, respectively) by the IRC.

\section{Results}

Of the 120 subjects enrolled in the study, all were randomized and received all planned treatments and thus formed the FAS. Of these 120 subjects, 115 subjects completed the primary endpoint visit (24-week visit; 3 were lost to follow-up and 2 were overdue for more than 2 weeks). All subjects completed the 36- and the 52-week visits; therefore, 120 subjects were included in the SA. Product allocation was balanced between right and left NLFs.

\section{Demographic and baseline characteristics}

Most subjects were female (96.67\%) with a mean age of 43 years (range 29-64 years) and a body mass index of 21.36 $\mathrm{m}^{2} / \mathrm{kg}$. One hundred nineteen out of 120 subjects were Han Chinese.

Of the 120 subjects, 102 had a symmetrical WSRS severity score of 3,16 had a symmetrical score of 4 , and 2 had a score of 3 in one NLF and 4 in the other (Table 1).

At initial treatment, local anesthesia was administered identically for each NLF. The injection dose was similar between groups; median total volume injected (initial plus touch-up) was $1.00 \mathrm{~mL}$ for both products (range: PV 0.3-2.0 $\mathrm{mL}$, RL 0.6-2.0 mL; Table 2).

\section{Primary endpoint: IRC assessment of improvement rates at 24 weeks}

The IRC-evaluated improvement rates using WSRS at 24 weeks (PP population) were $68.70 \%$ for PV and $52.17 \%$ for RL, resulting in a difference of $95 \%$ CI of $8.20 \%$, a value $<15 \%$. PV was shown to be statistically noninferior to RL.

Table I Demographic and baseline characteristics

\begin{tabular}{|l|l|}
\hline Characteristic & Description \\
\hline Age, years & \\
\hline Mean (range) & $43.07(29-64)$ \\
\hline Female, N (\%) & $\mathrm{I} 16(96.67)$ \\
\hline Nationality & \\
\hline Han Chinese & $\mathrm{N}=119$ \\
\hline Non-Han Chinese & $\mathrm{N}=1$ \\
\hline Medical history & \\
\hline WSRS severity score (I-5) & \\
\hline 3 symmetrical & $\mathrm{N}=102$ \\
\hline 4 symmetrical & $\mathrm{N}=16$ \\
\hline 4 and 3 & $\mathrm{N}=2$ \\
\hline
\end{tabular}

Abbreviation: WSRS, Wrinkle Severity Rating Scale.
Table 2 Treatment characteristics (safety population)

\begin{tabular}{|c|c|c|}
\hline Characteristics & PV & $\mathbf{R L}$ \\
\hline Initial treatment & $N=120$ & $\mathrm{~N}=120$ \\
\hline Touch-up treatment & $\mathrm{N}=\mathrm{I}$ & $\mathrm{N}=\mathrm{I}$ \\
\hline \multicolumn{3}{|c|}{ Injection volume, median (min, max) } \\
\hline Total, $\mathrm{mL}$ & $\mathrm{I} .00(0.3,2.0)$ & $1.00(0.6,2.0)$ \\
\hline
\end{tabular}

Abbreviations: PV, Princess ${ }^{\circledR}$ VOLUME; RL, Restylane ${ }^{\circledast}$.

Noninferiority was also established in the FAS population $(p=0.001)$.

At 24 weeks, $69.17 \%$ of subjects treated with PV and $53.33 \%$ of subjects treated with RL remained responders (Figure 1).

\section{Secondary endpoints: IRC and subject assessments of NLF score improvement over time}

GAIS improvement over time was more favorable for PV than for RL. IRC-assessed GAIS change over time showed that the mean scores for PV were lower than the mean scores for RL, with a significant difference at 4 weeks (2.02 vs 2.11; $p=0.012$ ) and at 24 weeks ( 1.93 vs $2.05 ; p=0.007$ ), for the FAS population, which remained similar for the PP population (Figure 2). Subject-assessed mean improvement rates based on GAIS scores for PV were lower than the scores for RL at all time points, but without significant difference (Figure 3).

\section{Safety}

Of the 120 subjects included in the SA population, 58 (48.33\%) reported at least one AE for PV, and 53 (44.17\%) reported at least one AE for RL (Table 3). Overall, the most frequently reported AEs for PV and RL were swelling (30.83\% and $29.17 \%$, respectively) and pain $(21.67 \%$ and $23.33 \%$, respectively). The incidence rates of AEs were similar in both groups. Overall, most AEs lasted for 14 days or less in $98.28 \%$ and $96.23 \%$ of subjects, who had a treatment site response to PV or RL, respectively.

Most of AEs related to PV and RL were mild (99.04\% and $97.06 \%$, respectively) in severity. All AEs associated with either product resolved without sequelae, and treatment with ice compression and massage was sufficient for the majority of AEs. There were no severe AEs in the clinical investigation.

\section{Discussion}

The objective of the present study was to compare the efficacy and safety of PV vs RL. The efficacy of PV treatment for correction of moderate-to-severe NLFs in Chinese subjects was statistically noninferior to RL treatment; thus, the primary 


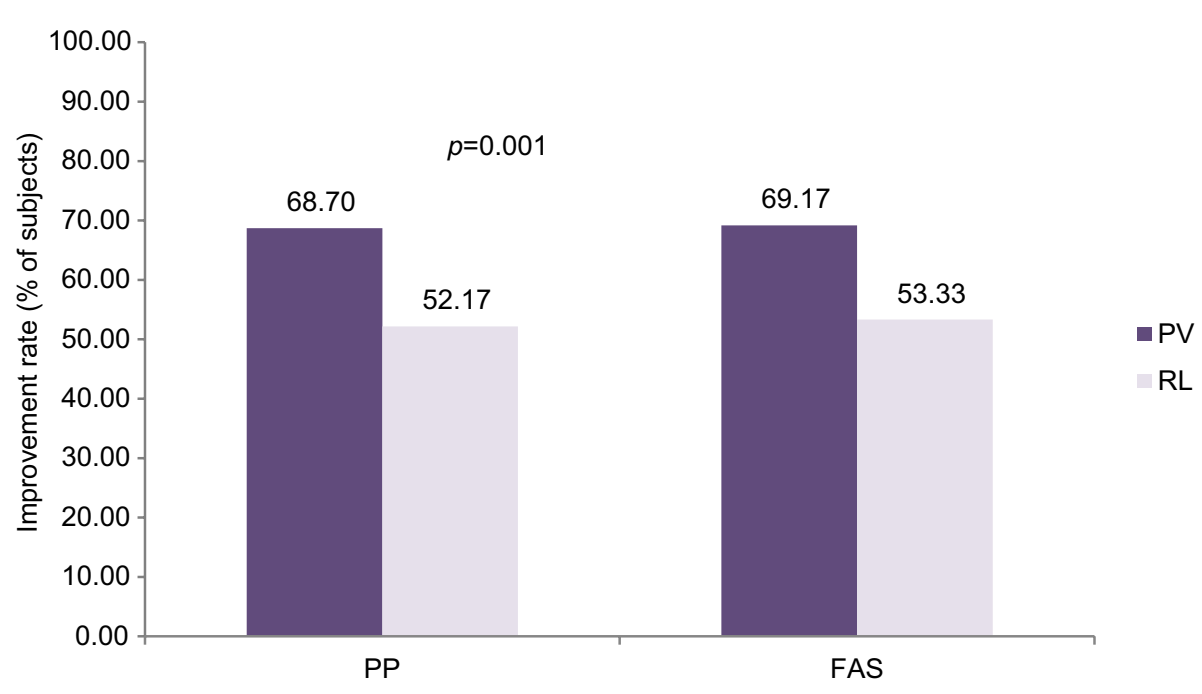

Figure I WSRS improvement rates assessed by the Independent Review Committee at 24 weeks.

Abbreviations: FAS, full analysis set; PP, per-protocol; PV, Princess ${ }^{\circledast}$ VOLUME; RL, Restylane ${ }^{\circledR}$; WSRS, Wrinkle Severity Rating Scale.

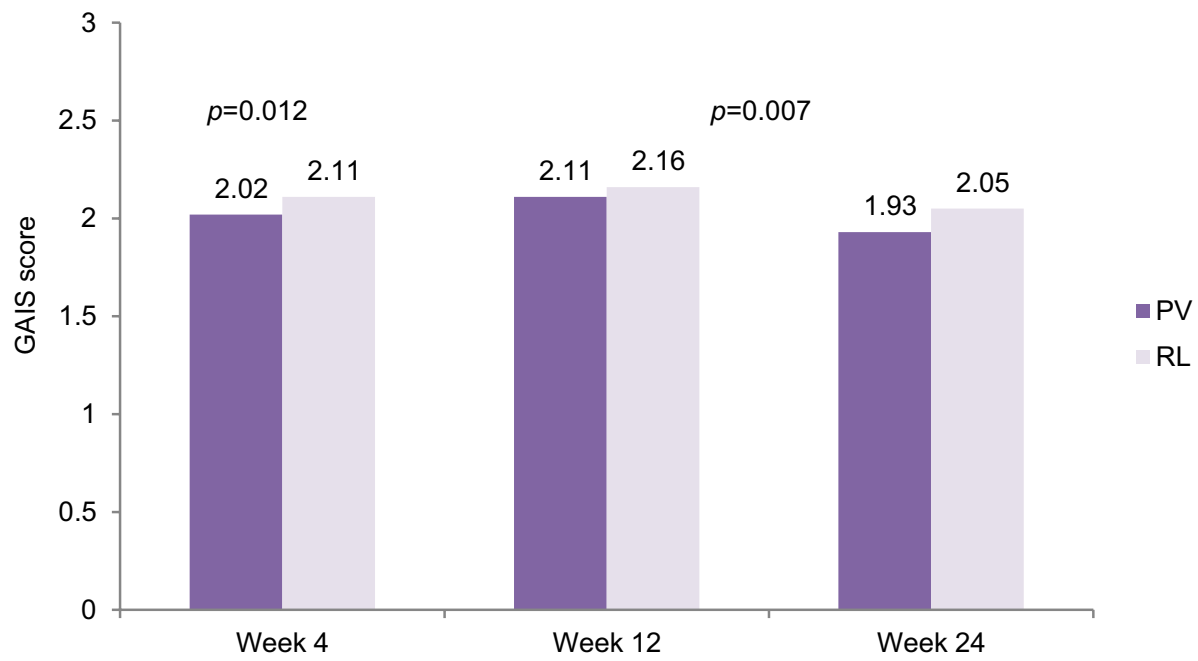

Figure 2 GAIS score assessed by the Independent Review Committee over time for the FAS population.

Abbreviations: FAS, full analysis set; GAIS, Global Aesthetic Improvement Scale; PV, Princess ${ }^{\circledR}$ VOLUME; RL, Restylane ${ }^{\circledR}$.

endpoint of this clinical investigation was successfully met. Correction was sustained through 24 weeks based on IRC assessment of improvement rates using the WSRS. The proportion of subjects with an improvement rate $\geq 1$ point for PV was comparable to or better than for RL at 24 weeks, as evaluated by the IRC. The efficacy of RL observed in this study was consistent with previous reports. ${ }^{12-14}$ In the study of Rzany et al, $78.8 \%$ of sides treated with RL had over 1 score improvement 6 months after injection. ${ }^{13}$

The mean GAIS scores for PV, as assessed by IRC and by subjects, were lower than those for RL at all time points, which are consistent with the result of WSRS improvement scores. These results indicate that PV is effective in reducing the severity of NLFs.
The median values were $1.00 \mathrm{~mL}$ in both treatment groups. The volume injected was chosen by the investigator and depended on the depth of NLF to be corrected. Median volumes used in the current investigation were similar to volumes used in the RL efficacy study in Chinese subjects for correction of severe NLFs $(1.00 \mathrm{~mL}) .^{10,11}$

Secondary endpoints included evaluation of WSRS improvement rate, WSRS improvement scores, and GAIS scores of subjects at weeks 4, 12, and 24, injection dose and frequency, and 2 point (or higher) improvements in WSRS scores. Evaluation of the secondary endpoints confirmed that the efficacy of PV is comparable to, or even better than, RL.

PV and RL had similar safety profiles in the current investigation. The AEs reported by subjects included ery- 


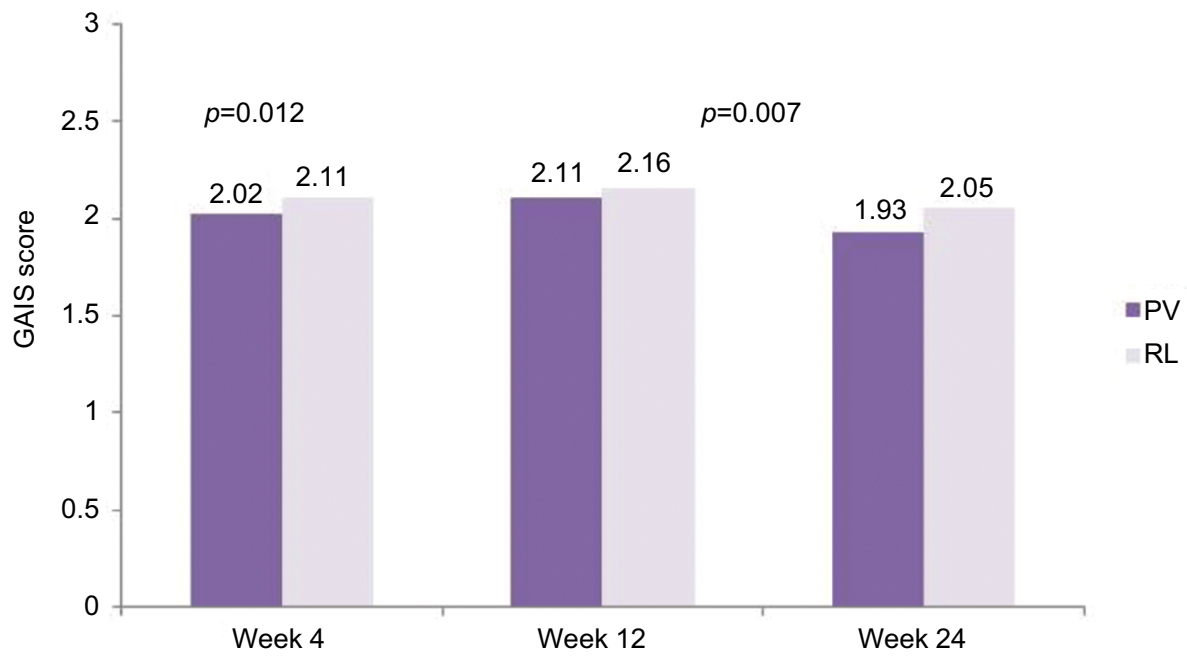

Figure 3 GAIS score assessed by the subjects over time for the FAS population.

Abbreviations: FAS, full analysis set; GAIS, Global Aesthetic Improvement Scale; PV, Princess ${ }^{\circledR}$ VOLUME; RL, Restylane ${ }^{\circledR}$.

Table 3 Incidence of treatment site AEs

\begin{tabular}{|c|c|c|}
\hline AE & PV & $\mathbf{R L}$ \\
\hline \multicolumn{3}{|c|}{ Subjects comprised in the safety assessment population $(n=120)$} \\
\hline Subjects with any event, N (\%) & $58(48.33)$ & $53(44.17)$ \\
\hline \multicolumn{3}{|l|}{ Type, N (\%) } \\
\hline Swelling & $37(30.83)$ & $35(29.17)$ \\
\hline Pain & $26(21.67)$ & $28(23.33)$ \\
\hline Injection site hemorrhage & $14(\mathrm{II} .67)$ & $15(12.50)$ \\
\hline Injection site nodules & $20(16.67)$ & $13(10.83)$ \\
\hline Postoperative wound complications & $3(2.5)$ & $4(3.33)$ \\
\hline Wound complication & $\mathrm{I}(0.83)$ & $\mathrm{I}(0.83)$ \\
\hline Postprocedural hemorrhage & $0(0.00)$ & $2(1.67)$ \\
\hline
\end{tabular}

Abbreviations: AE, adverse event; PV, Princess ${ }^{\circledR}$ VOLUME; RL, Restylane ${ }^{\circledR}$.

thema, swelling, pain, bruising, nodule, pruritus, tenderness, hemorrhage and others, which are the AEs most commonly reported after treatment with dermal fillers. Several reports on HA with RL reported the filler to be safe and well tolerated, with the majority of the subjects having transient and mild injection site reactions, even up to 12 months, follow-up. ${ }^{13,15,16}$

PV and RL achieved the best NLF correction effect at 4 weeks, as measured by the WSRS. Analytical results of mean GAIS score changes were in agreement with these results.

\section{Conclusion}

This clinical investigation demonstrated that PV is safe and effective in correction of moderate-to-severe NLFs in Chinese subjects. No new safety signals were seen in this population compared with non-Asian subjects, indicating a similar and favorable risk-benefit profile. Additionally, the treatment with PV in this study demonstrated noninferiority to RL treatment $(10 \%$ and $15 \%$ noninferiority was demonstrated with $95 \% \mathrm{CI}$ ) and consistently better response across all evaluation parameters and time points.

\section{Acknowledgments}

The authors would like to Thank Ira Lawrence, $\mathrm{PhD}$, for his contribution to this study. This study was sponsored by Qualtech Consulting Corporation, Xicheng District, Beijing, China.

\section{Disclosure}

$\mathrm{XD}$ and LL have been principal investigators in the clinical study. SH, ZIS, ABZ and MP are employees of CROMAPHARMA. WP and RRB are past employees of CROMAPHARMA. The authors report no other conflicts of interest in this work.

\section{References}

1. John HE, Price RD. Perspectives in the selection of hyaluronic acid fillers for facial wrinkles and aging skin. Patient Prefer Adherence. 2009;3:225-230.

2. Bogdan Allemann I, Baumann L. Hyaluronic acid gel (Juvéderm) preparations in the treatment of facial wrinkles and folds. Clin Interv Aging. 2008;3(4):629-634.

3. Fakhari A, Berkland C. Applications and emerging trends of hyaluronic acid in tissue engineering, as a dermal filler and in osteoarthritis treatment. Acta Biomater. 2013;9(7):7081-7092.

4. Beasley K, Weiss M, Weiss R. Hyaluronic acid fillers: a comprehensive review. Facial Plast Surg. 2009;25(02):86-94.

5. Raspaldo H. Volumizing effect of a new hyaluronic acid sub-dermal facial filler: a retrospective analysis based on 102 cases. J Cosmet Laser Ther. 2008;10(3):134-142.

6. American Society for Aesthetic Plastic Surgery. Statistics. 2016. Available from: https://www.surgery.org/sites/default/files/ASAPSStats2016.pdf. Accessed November 17, 2018.

7. Day DJ, Littler CM, Swift RW, Gottlieb S. The wrinkle severity rating scale. Am J Clin Dermatol. 2004;5(1):49-52. 
8. Narins RS, Brandt F, Leyden J, Lorenc ZP, Rubin M, Smith S. A randomized, double-blind, multicenter comparison of the efficacy and tolerability of Restylane versus Zyplast for the correction of nasolabial folds. Dermatol Surg. 2003;29(6):588-595.

9. Liu JP, Hsueh HM, Hsieh E, Chen JJ. Tests for equivalence or noninferiority for paired binary data. Stat Med. 2002;21(2):231-245.

10. Li D, Xie Y, Li Q, et al. Safety and effectiveness of Juvéderm Ultra Plus injectable gel in correcting severe nasolabial folds in Chinese subjects. Plast Reconstr Surg Glob Open. 2017;5(1):e1133.

11. Narins RS, Dayan SH, Brandt FS, Baldwin EK. Persistence and improvement of nasolabial fold correction with nonanimal-stabilized hyaluronic acid 100,000 gel particles/ $\mathrm{mL}$ filler on two retreatment schedules: results up to 18 months on two retreatment schedules. Dermatol Surg. 2008;34 (Suppl 1):S2-S8.

12. Nast A, Reytan N, Hartmann V. Efficacy and durability of two hyaluronic acid-based fillers in the correction of nasolabial folds: results of a prospective, randomized, double-blind, actively controlled clinical pilot study. Dermatol Surg. 2011;37(6):768-775.
13. Rzany B, Bayerl C, Bodokh I, et al. Efficacy and safety of a new hyaluronic acid dermal filler in the treatment of moderate nasolabial folds: 6-month interim results of a randomized, evaluator-blinded, intra-individual comparison study. J Cosmet Laser Ther. 2011;13(3): 107-112.

14. Suh JH, Oh CT, Im SI, Lim JS, Kim BJ, Lee JH. A multicenter, randomized, double-blind clinical study to evaluate the efficacy and safety of a new monophasic hyaluronic acid filler with lidocaine $0.3 \%$ in the correction of nasolabial fold. J Cosmet Dermatol. 2017;16(3): 327-332.

15. Ascher B, Bayerl C, Brun P, et al. Efficacy and safety of a new hyaluronic acid dermal filler in the treatment of severe nasolabial lines - 6-month interim results of a randomized, evaluator-blinded, intra-individual comparison study. J Cosmet Dermatol. 2011;10(2):94-98.

16. Ascher B, Bayerl C, Kestemont P, Rzany B, Edwartz C, Podda M. A 12-month follow-up, randomized comparison of effectiveness and safety of two hyaluronic acid fillers for treatment of severe nasolabial folds. Dermatol Surg. 2017;43(3):389-395.
Clinical, Cosmetic and Investigational Dermatology

\section{Publish your work in this journal}

Clinical, Cosmetic and Investigational Dermatology is an international, peer-reviewed, open access, online journal that focuses on the latest clinical and experimental research in all aspects of skin disease and cosmetic interventions. This journal is included on PubMed. The manuscript management system is completely online 